\title{
Knockdown of NCOA5 suppresses viability, migration and epithelial-mesenchymal transition, and induces adhesion of breast cancer cells
}

\author{
YANFANG TAN ${ }^{1}$, FUHUI LIU $^{2}$ and PEI XU ${ }^{3}$ \\ ${ }^{1}$ Department of Clinical Laboratory, People's Hospital of Deyang City, Deyang, Sichuan 618000; \\ ${ }^{2}$ Department of Blood Transfusion, Affiliated Hospital of Zunyi Medical College, Zunyi, Guizhou 563000; \\ ${ }^{3}$ Department of Pathology, People's Hospital of Deyang City, Deyang, Sichuan 618000, P.R. China
}

Received February 6, 2021; Accepted April 27, 2021

DOI: $10.3892 / \mathrm{ol} .2021 .12955$

\begin{abstract}
Nuclear receptor coactivator 5 (NCOA5) has been reported to be involved in the regulation of several malignancies; however, to the best of our knowledge, its role in breast cancer is still unknown. The present study aimed to reveal the biological function of NCOA5 in breast cancer cells. NCOA5 expression in breast cancer tissues and cell lines was examined using reverse transcription-quantitative PCR and western blotting. Small interfering RNA (siRNA) against NCOA5 (siNCOA5) was transfected into MDA-MB-453 and MCF-7 cells to knock down NCOA5. MTT, transwell migration and cell adhesion assays were performed to determine cell viability, migration and adhesion abilities of breast cancer cells, respectively. In addition, the expression levels of $\mathrm{N}$-cadherin, Vimentin and E-cadherin were examined by western blotting. It was revealed that NCOA5 expression was significantly increased in breast cancer tissues and cell lines. Knockdown of NCOA5 suppressed breast cancer cell viability and migration, and induced cell adhesion. Compared with those in cells transfected with non-targeting negative control siRNA, the protein expression levels of $\mathrm{N}$-cadherin and Vimentin were significantly decreased, whereas the protein expression levels of E-cadherin were significantly increased in cells transfected with siNCOA5. The present study demonstrated that knockdown of NCOA5 suppressed cell viability and migration, induced cell adhesion, and inhibited epithelial-mesenchymal transition of breast cancer cells, indicating that NCOA5 may serve a tumor-promoting role in breast cancer.
\end{abstract}

Correspondence to: Dr Yanfang Tan, Department of Clinical Laboratory, People's Hospital of Deyang City, Section 1, 173 Taishan North Road, Deyang, Sichuan 618000, P.R. China

E-mail: tyf1329500486@163.com

Key words: nuclear receptor coactivator 5, breast cancer, epithelial-mesenchymal transition

\section{Introduction}

Breast cancer is the most common malignancy in women and the second leading cause of cancer-associated mortality in women worldwide (1). Patients with early-stage breast cancer can be cured (2). Early diagnosis and early treatment of breast cancer are the key to improving its prognosis (3). Despite marked advances in its treatment, breast cancer remains a major health problem (4).

Nuclear receptor coactivator 5 (NCOA5) is a nuclear receptor coregulator, which interacts with $\alpha$ and $\beta$ estrogen receptors, as well as an orphan nuclear receptor (nuclear receptor subfamily 1 group D member 2), independently of the AF2 domain (5). NCOA5 is considered to have both coactivator and corepressor functions, and could modulate estrogen receptor $\alpha$-mediated transcription (6). In addition, NCOA5 interacts with tumor suppressor Tat-interacting protein $(30 \mathrm{kDa})$ and regulates c-myc transcription (7). NCOA5 has been reported to be involved in the regulation of several malignancies, such as hepatocellular carcinoma, colorectal cancer and papillary thyroid cancer (8-14). Ye et al (15) reported that NCOA5 expression was increased in luminal breast cancer tissues, and high NCOA5 expression was associated with the progression and prognosis of patients with luminal breast cancer. However, to the best of our knowledge, the mechanisms underlying the role of NCOA5 in breast cancer are still unknown.

In the present study, the expression levels of NCOA5 in breast cancer tissues and a series of breast cancer cell lines were detected. The effects of NCOA5 on the viability, migration, adhesion and epithelial-mesenchymal transition (EMT) of breast cancer cells were evaluated using loss-of-function experiments. The present study aimed to reveal the biological function of NCOA5 in breast cancer, and provided a potential therapeutic target for patients with breast cancer.

\section{Materials and methods}

Tissue collection. Breast cancer tissues and adjacent normal tissues (distance from tumor margin, $2 \mathrm{~cm}$ ) were collected from 25 female patients with breast cancer who underwent surgical resection at the People's Hospital of Deyang City 
(Deyang, China). These patients were recruited between January 2019 and November 2020. The patients were aged 35-67 years with a mean age of 51.3 years, and all patients had not been treated before surgical resection. The inclusion criteria were: i) Female subjects aged 18-70 years; ii) histology or cytology confirmed breast cancer; iii) ECOG score was 0-1; iv) the expected survival time of the case was $>3$ months; v) left ventricular ejection fraction $\geq 55 \%$; vi) laboratory examination data met the following criteria: Hemoglobin $\geq 90 \mathrm{~g} / \mathrm{l}$, absolute neutrophil count $\geq 1.5 \times 10^{9} / 1$, platelets $\geq 100 \times 10^{9} / 1$, total bilirubin $\leq 1.5$ times upper limit of normal (x ULN), aspartate transaminase/alanine transaminase $\leq 2.5 \mathrm{x}$ ULN or $\leq 5 \mathrm{x}$ ULN in case of liver metastasis, creatinine $\leq 1.5 \mathrm{x}$ ULN and international normalized ratio $\leq 1.5 \mathrm{x}$ ULN, partial thromboplastin time $\leq 1.5 \mathrm{x}$ ULN; and vii) volunteered to participate in the research and signed the informed consent form. The exclusion criteria were: i) Patients with heart, liver, kidney and hematopoietic system diseases; ii) brain metastasis; iii) with double or multiple cancers; iv) suffering from clinically significant active, acute, chronic infection or bleeding; v) hypertension is not under control; vi) pregnant or lactating women, mental disorders; vii) participate in any other clinical trials within 1 month before enrollment; viii) received therapy before surgery; and ix) the researcher judged that the subjects had any other conditions that were not suitable for the trial. The study was approved by the Ethics Committee of the People's Hospital of Deyang City (Deyang, China). All patients signed an informed consent form prior to enrollment in the present study.

Cell culture and transfection. The human MCF-10A normal breast epithelial cell line and human breast cancer cell lines (MDA-MB-231, MDA-MB-453 and SK-BR-3 breast adenocarcinoma cell lines, and MCF-7 invasive ductal carcinoma cell line) were purchased from The Cell Bank of Type Culture Collection of The Chinese Academy of Sciences. The cells were cultured in DMEM (Thermo Fisher Scientific, Inc.) supplemented with $10 \%$ FCS (Gibco; Thermo Fisher Scientific, Inc.), $100 \mathrm{U} / \mathrm{ml}$ penicillin (Gibco; Thermo Fisher Scientific, Inc.) and $100 \mu \mathrm{g} / \mathrm{ml}$ streptomycin (Gibco; Thermo Fisher Scientific, Inc.) in a humidified atmosphere with $5 \% \mathrm{CO}_{2}$ at $37^{\circ} \mathrm{C}$. The small interfering RNA (siRNA/si) directly against human NCOA5 (siNCOA5) and the non-targeting negative control siRNA (siNC) were purchased from Shanghai GenePharma Co., Ltd. The sequences of siRNAs were as follows: siNCOA5 sense, 5'-AGGGAUCUUAGAGACUUUCGUTT-3' and antisense, 5'-ACGAAAGUCUCUAAGAUCCCUTT-3'; siNC sense, 5'-UUCUCCGAACGUGUCACGUTT-3' and antisense, 5'-ACG UGACACGUUCGGAGAATT-3'. Cells $\left(5 \times 10^{5}\right.$ cells/ml) were plated into the wells and transfected with $50 \mathrm{nM}$ siRNA using Lipofectamine ${ }^{\circledR} 3000$ transfection reagent (Invitrogen; Thermo Fisher Scientific, Inc.) at $37^{\circ} \mathrm{C}$ for $6 \mathrm{~h}$, according to the manufacturer's protocols. At $48 \mathrm{~h}$ after transfection, cells were collected for subsequent experiments.

Reverse transcription-quantitative PCR (RT-qPCR). Total RNA was isolated from tissues or cells using TRIzol ${ }^{\circledR}$ (Invitrogen; Thermo Fisher Scientific, Inc.). The isolated RNA was then reverse transcribed into cDNA using a Prime-Script ${ }^{\circledR}$ RT reagent kit (Takara Biotechnology Co., Ltd.) according to the manufacturer's protocol. The primers used in the present study were as follows: NCOA5 forward, 5'-CAAGTGCTCCCCTCTGC TAC-3' and reverse, 5'-CTGTTTGCTGCTGTGGAAAA-3'; GAPDH forward, 5'-CGACCACTTTGTCAAGCTCA-3' and reverse, 5'-AGGGGTCTACATGGCAACTG-3'. The specific reaction procedure was: $42^{\circ} \mathrm{C}$ for $15 \mathrm{~min}, 85^{\circ} \mathrm{C}$ for $5 \mathrm{sec}$, and $4^{\circ} \mathrm{C}$ until further use. The cDNA was used as a template for $\mathrm{qPCR}$. The PCR reaction was performed with the SYBR Green PCR Master Mix (Quantabio) on an Applied Biosystems 7900HT Fast Real-Time PCR System (Applied Biosystems; Thermo Fisher Scientific, Inc.). Amplification was performed using the following conditions: Initial denaturation at $95^{\circ} \mathrm{C}$ for $1 \mathrm{~min}$, followed by 40 cycles of $95^{\circ} \mathrm{C}$ for $5 \mathrm{sec}$ and $60^{\circ} \mathrm{C}$ for $20 \mathrm{sec}$. GAPDH was used as a reference gene. Relative gene expression of NCOA5 was calculated using the $2^{-\Delta \Delta \mathrm{Cq}}$ method (16).

Western blotting. Proteins were extracted from the tissues or cells using RIPA Lysis Buffer (EMD Millipore). A BCA Protein Assay Kit (Pierce; Thermo Fisher Scientific, Inc.) was used to determine the protein concentration of lysates. A total of $50 \mu \mathrm{g}$ protein per lane was separated via 10\% SDS-PAGE, and the protein was then transferred to a polyvinylidene difluoride membrane (EMD Millipore). Next, the membrane was blocked with $10 \%$ skim milk at $4^{\circ} \mathrm{C}$ overnight, followed by incubation at $37^{\circ} \mathrm{C}$ for $2 \mathrm{~h}$ with the following antibodies: NCOA5 (cat. no. ab70831; dilution, 1:500), N-cadherin (cat. no. ab18203; dilution, 1:1,000), Vimentin (cat. no. ab45939; dilution, 1:800), E-cadherin (cat. no. ab212059; dilution, 1:800) and GAPDH (cat. no. ab9485; dilution, 1:2,000). All these antibodies were purchased from Abcam. After washing three times with PBS with $0.05 \%$ Tween-20 at room temperature for $5 \mathrm{~min}$ each, the membrane was incubated with the Goat Anti-Rabbit IgG H\&L (HRP) antibody (cat. no. ab6721; dilution, 1:2,000; Abcam) at $37^{\circ} \mathrm{C}$ for $1 \mathrm{~h}$. Finally, the membrane was treated with ECL Plus Western Blotting Substrate (Thermo Fisher Scientific, Inc.) and the band density was determined by densitometric analysis (Image Lab v4.0; Bio-Rad Laboratories, Inc.).

MTT. To determine cell viability, a total of $1.5 \times 10^{4}$ cells were seeded in each well of 96-well plates. The cells were cultured in DMEM supplemented with $10 \% \mathrm{FCS}$ at $37^{\circ} \mathrm{C}$ in a humidified atmosphere of $5 \% \mathrm{CO}_{2}$ for $24,48,72$ and $96 \mathrm{~h}$. Cell viability was determined using a MTT Kit (Beyotime Institute of Biotechnology) according to the manufacturer's protocol. Cells were incubated with MTT solution at $37^{\circ} \mathrm{C}$ for $4 \mathrm{~h}$, and then dimethyl sulfoxide (Sigma-Aldrich; Merck KGaA) was added to dissolve the formazan crystals. Absorbance was detected at a wavelength of $490 \mathrm{~nm}$ using a microplate reader (Bio-Rad Laboratories, Inc.).

Transwell migration assay. Cell migration was analyzed using 6-well transwell insert chambers with a pore size of $8 \mu \mathrm{m}$ (Corning, Inc.). The cells were suspended in DMEM without FCS and then $5 \times 10^{5}$ cells were cultured in the upper chamber. DMEM supplemented with $10 \%$ FCS was added to the bottom chamber as a chemoattractant. Following incubation at $37^{\circ} \mathrm{C}$ for $24 \mathrm{~h}$, the migrated cells were fixed with $95 \%$ ethanol for $15 \mathrm{~min}$ at $37^{\circ} \mathrm{C}$. Subsequently, the cells were stained with $0.3 \%$ hematoxylin (Leagene Biotechnology) for $15 \mathrm{~min}$ at $37^{\circ} \mathrm{C}$. Cells were observed under a light microscope (Nikon Corporation), and the number of migrated cells was calculated for five random fields. 

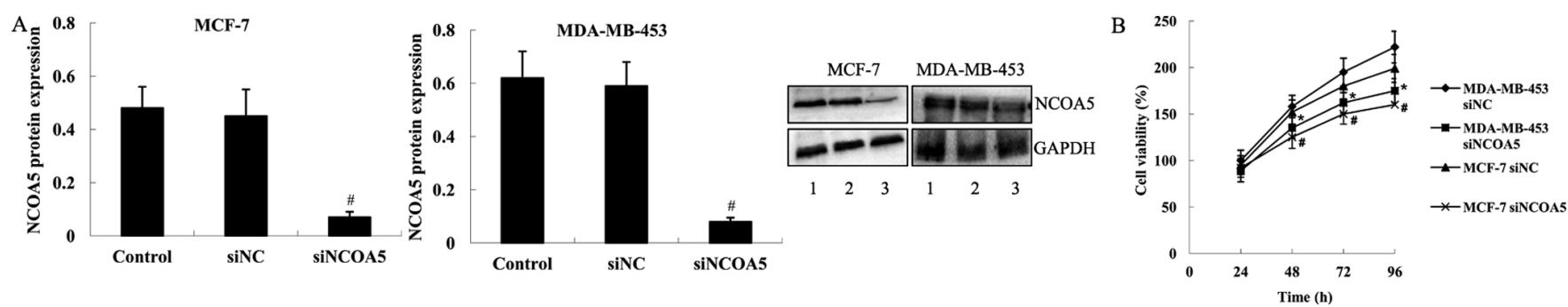

Figure 2. Effect of NCOA5 on the viability of breast cancer cells. (A) NCOA5 expression in MCF-7 and MDA-MB-453 cells following transfection with siNCOA5. Lane 1, control; lane 2, siNC; lane 3, siNCOA5. "P<0.01 vs. siNC. (B) Effect of siNCOA5 on the viability of MCF-7 and MDA-MB-453 cells. ${ }^{*} \mathrm{P}<0.05$ vs. MDA-MB-453 siNC; ${ }^{\text {P }}<<0.05$ vs. MCF-7 siNC. NCOA5, nuclear receptor coactivator 5; si, small interfering RNA; NC, negative control.
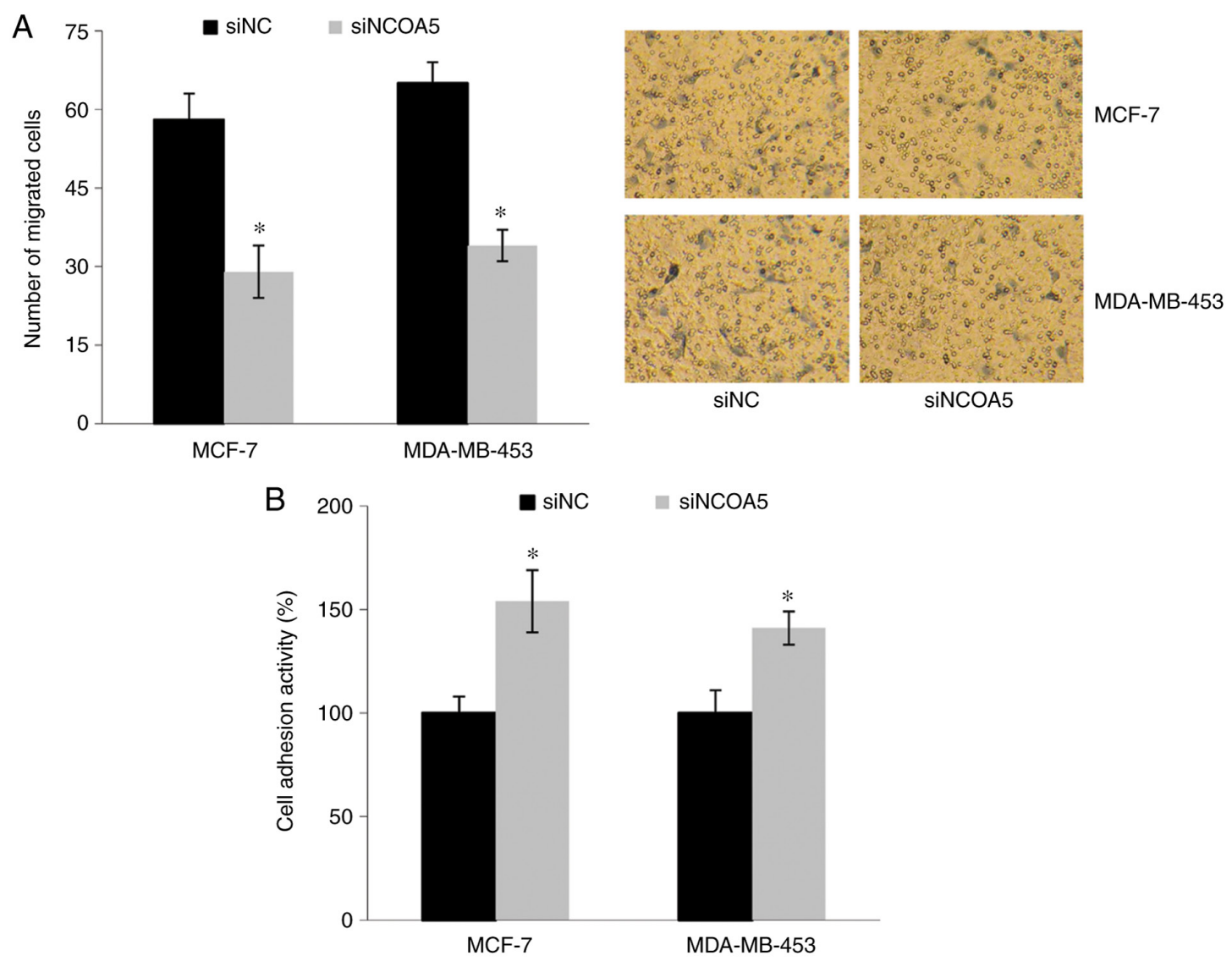

Figure 3. Effect of NCOA5 on the migration and adhesion of breast cancer cells. (A) Effect of NCOA5 on cell migration. Magnification, x200. (B) Effect of NCOA5 on cell adhesion. ${ }^{*} \mathrm{P}<0.05$ vs. siNC. NCOA5, nuclear receptor coactivator 5; si, small interfering RNA; NC, negative control.

Effect of NCOA5 on the migration and adhesion abilities of breast cancer cells. Transwell migration assays were performed to investigate the effect of NCOA5 on cell migration. As shown in Fig. 3A, the number of migrated cells was significantly decreased in the siNCOA5 group compared with the siNC group. Furthermore, a cell adhesion assay revealed that the adhesion abilities of MDA-MB-453 and MCF-7 cells were significantly increased following transfection with siNCOA5 (Fig. 3B).

Effect of NCOA5 on EMT of breast cancer cells. The protein expression levels of $\mathrm{N}$-cadherin, Vimentin and $\mathrm{E}$-cadherin were examined by western blot analysis to investigate the effect of NCOA5 on EMT of breast cancer cells. Compared with those in the cells transfected with siNC, the protein expression levels of $\mathrm{N}$-cadherin and Vimentin were significantly decreased, whereas the protein expression levels of E-cadherin were significantly increased in MDA-MB-453 cells transfected with siNCOA5. In MCF-7 cells, the expression levels of $\mathrm{N}$-cadherin and Vimentin were significantly decreased, and E-cadherin expression was significantly increased in siNCOA5-tranfected cells compared with siNC-transfected cells (Fig. 4).

\section{Discussion}

NCOA5 is a unique nuclear receptor coactivator with both co-activation and co-repression functions (6). The abnormal expression of estrogen receptor is associated with various types of cancer (17), and previous studies have demonstrated 

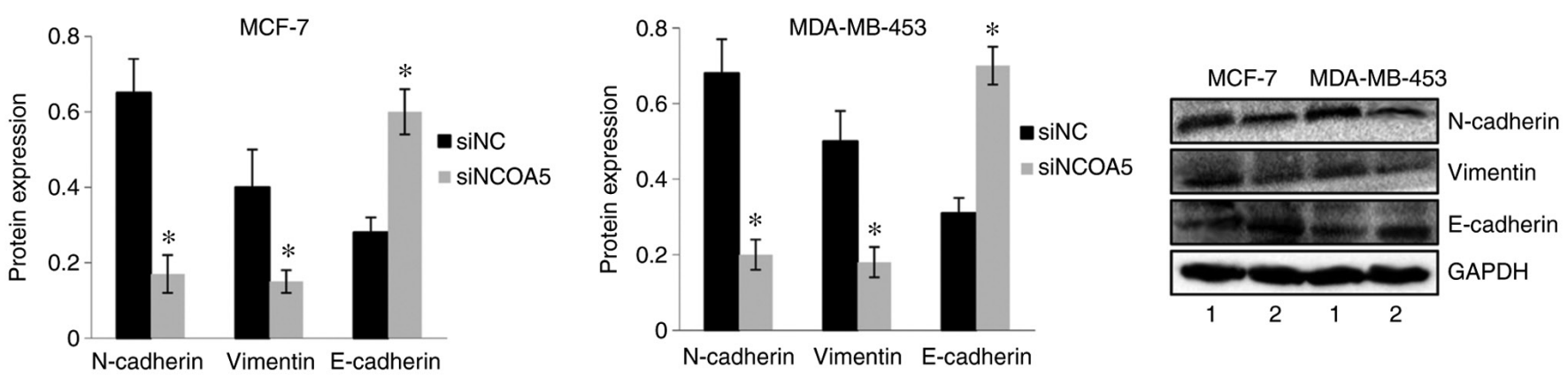

Figure 4. Effect of NCOA5 on the expression levels of epithelial-mesenchymal transition-related proteins in breast cancer cells. Lane 1, siNC; lane 2, siNCOA5. ${ }^{*} \mathrm{P}<0.05$ vs. siNC. NCOA5, nuclear receptor coactivator 5; si, small interfering RNA; NC, negative control.

that NCOA5 can regulate estrogen receptor-mediated transcription in human cells (5-7). The role of NCOA5 in human cancer has attracted increasing attention. Abnormal expression of NCOA5 has been reported in a variety of tumors, including esophageal squamous cell carcinoma, hepatocellular carcinoma and cervical cancer $(15,18-21)$. The present study demonstrated that NCOA5 expression was significantly increased in human breast cancer tissues compared with adjacent normal tissues. Furthermore, NCOA5 expression was significantly increased in breast cancer cell lines compared with a normal breast epithelial cell line. The present results were consistent with a previous report showing that NCOA5 was upregulated in luminal breast cancer tissues compared with adjacent normal tissues both in a validated cohort and The Cancer Genome Atlas cohort (15). Additionally, upregulation of NCOA5 has been identified in colorectal cancer (13). Conversely, NCOA5 has been reported to be downregulated in hepatocellular carcinoma, cervical cancer, esophageal squamous cell carcinoma, papillary thyroid carcinoma and osteosarcoma (18-21). These reports suggest that alterations of NCOA5 are involved in the carcinogenesis and progression of human cancer.

Accumulating evidence has demonstrated that NCOA5 serves as a tumor suppressor or an oncogene in different tumor types (12-14,19). For example, NCOA5 expression is associated with the clinicopathological features of patients with colorectal cancer (13). Knockdown of NCOA5 markedly suppresses the proliferation, migration and invasion of colorectal cancer cells, induces cell cycle G1 phase arrest, and inhibits in vivo xenograft growth of colorectal cancer cells (13). Similarly, knockout of NCOA5 inhibits proliferation and migration in hepatocellular carcinoma cells (12). By contrast, Zheng et al (14) reported that NCOA5 is a tumor suppressor gene in papillary thyroid carcinoma, and reduced NCOA5 expression is associated with the aggressive clinicopathological features of patients with papillary thyroid carcinoma. Additionally, a study revealed that low NCOA5 expression predicts poor prognosis in human cervical cancer, and downregulation of NCOA5 results in an increase in proliferation, migration and invasion of HeLa cells (19). Ye et al (15) demonstrated that high NCOA5 expression was an independent high risk factor in luminal breast cancer, and patients with high NCOA5 expression had a lower overall survival rate. Yuan et al (22) reported that NCOA5 expression could be stimulated by methionine and leucine in bovine mammary epithelial cells; however, phosphatidylinositol 3-kinase inhibition could abolish the stimulatory effect of methionine and leucine on NCOA5. NCOA5 can bind to the mTOR promoter, and induce mTOR phosphorylation and $\beta$-casein synthesis (22). However, the precise role and the cellular mechanism for NCOA5 in breast cancer are still largely unknown. The present study explored the biological function of NCOA5 in vitro. NCOA5 expression was significantly increased in breast cancer cell lines. Therefore, loss-of-function experiments were performed in MDA-MB-453 and MCF-7 cell lines, which exhibited higher expression levels of NCOA5 than MDA-MB-231 and SK-BR-3 cell lines. It was demonstrated that knockdown of NCOA5 suppressed the viability and migration, and induced adhesion of breast cancer cells, indicating that NCOA5 may act as a novel oncogene to promote the progression of breast cancer. The present finding was consistent with previous studies demonstrating that NCOA5 acts as an oncogene in colorectal cancer and hepatocellular carcinoma $(12,13)$. However, in papillary thyroid carcinoma and cervical cancer, NCOA5 acts as a tumor suppressor $(14,19)$. It was hypothesized that the role of NCOA5 in human cancer may be dependent on the tissue type. The mechanisms underlying the biological effects of NCOA5 in different types of human cancer should be further explored.

The present study further demonstrated that NCOA5 knockdown decreased the expression levels of $\mathrm{N}$-cadherin and Vimentin, and increased the expression levels of E-cadherin. It has been demonstrated that $\mathrm{N}$-cadherin, Vimentin and E-cadherin are key mediators of EMT (23-25). EMT is an important process for epithelial cancer metastasis (26-28). The loss of E-cadherin expression or gain of $\mathrm{N}$-cadherin and Vimentin expression contributes to EMT $(25,29,30)$. The present results supported the hypothesis that NCOA5 induced EMT in breast cancer cells, thus promoting tumor cell migration and invasion. Additional functional experiments are required to demonstrate the effects of the EMT phenotype of the cells.

Interestingly, the present findings suggested that NCOA5 could promote proliferation and aggressiveness of breast cancer cells. However, among all the breast cancer cell lines examined, the expression levels of NCOA5 were high in the least aggressive MCF-7 cell line (31). At present, there are few studies regarding the role of NCOA5 in breast cancer $(15,22)$, and the association between NCOA5 expression and the aggressiveness of breast cancer cell lines is still unclear. 
Whether the expression levels of NCOA5 could be used to predict the aggressiveness of breast cancer cell lines requires further investigation.

A limitation of the present study was that loss-of-function experiments were performed only in breast cancer cells. More studies on MCF-10A normal breast epithelial cell line are required to provide a negative control for the experiments performed using the breast cancer cell lines. Another limitation of the present study was that it only used an MTT assay to determine cell viability. Other assays are required to further examine the effect of NCOA5 on cell proliferation.

In conclusion, the present study demonstrated that NCOA5 expression was upregulated in human breast cancer tissues and breast cancer cell lines. Furthermore, the present study revealed the cellular mechanisms for NCOA5 in breast cancer, and demonstrated that knockdown of NCOA5 suppressed cell viability and migration, induced cell adhesion, and inhibited EMT of breast cancer cells, indicating that NCOA5 serves a tumor-promoting role in the progression of breast cancer. The present study suggested NCOA5 as a novel target for the treatment of breast cancer.

\section{Acknowledgements}

Not applicable.

\section{Funding}

No funding was received.

\section{Availability of data and materials}

The datasets used and/or analyzed during the current study are available from the corresponding author on reasonable request.

\section{Authors' contributions}

YT and FL designed the study, prepared the manuscript, and confirmed the authenticity of all the raw data. YT, FL and PX conducted the experiments. All authors were substantially involved in the research, acquisition of data, analysis and manuscript preparation. All authors read and approved the final manuscript.

\section{Ethics approval and consent to participate}

The present study was approved by the Ethics Committee of the People's Hospital of Deyang City (Deyang, China). All patients signed an informed consent form prior to enrollment in the present study.

\section{Patient consent for publication}

All patients signed an informed consent for enrollment and publication.

\section{Competing interests}

The authors declare that they have no competing interests.

\section{References}

1. Januškevičienė I and Petrikaitė V: Heterogeneity of breast cancer: The importance of interaction between different tumor cell populations. Life Sci 239: 117009, 2019.

2. Wörmann B: Breast cancer: Basics, screening, diagnostics and treatment. Med Monatsschr Pharm 40: 55-64, 2017.

3. Zeng Y, Zhang J, Meng J, Numthuam S and Naruse K: Application of multi-modal imaging mediated by iron carbon nanoparticles based on reinforcement learning in the diagnosis of breast nodules. J Nanosci Nanotechnol 21: 1154-1160, 2021.

4. Anastasiadi Z, Lianos GD, Ignatiadou E, Harissis HV and Mitsis M: Breast cancer in young women: An overview. Updates Surg 69: 313-317, 2017.

5. Zhang Z and Teng CT: Estrogen receptor alpha and estrogen receptor-related receptor alpha1 compete for binding and coactivator. Mol Cell Endocrinol 172: 223-233, 2001.

6. Sauvé F, McBroom LD, Gallant J, Moraitis AN, Labrie F and Giguère V: CIA, a novel estrogen receptor coactivator with a bifunctional nuclear receptor interacting determinant. Mol Cell Biol 21: 343-353, 2001.

7. Jiang C, Ito M, Piening V, Bruck K, Roeder RG and Xiao H: TIP30 interacts with an estrogen receptor alpha-interacting coactivator CIA and regulates c-myc transcription. J Biol Chem 279: 27781-27789, 2004.

8. Liu CY and Feng GS: NCOA5, a molecular link between type 2 diabetes and liver cancer. Hepatobiliary Surg Nutr 3: 106-108, 2014.

9. Liu X, Liu F, Gao S, Reske J, Li A, Wu CL, Yang C, Chen F, Luo R and Xiao H: A single non-synonymous NCOA5 variation in type 2 diabetic patients with hepatocellular carcinoma impairs the function of NCOA5 in cell cycle regulation. Cancer Lett 391: 152-161, 2017.

10. Dhar D, Seki E and Karin M: NCOA5, IL-6, type 2 diabetes, and HCC: The deadly quartet. Cell Metab 19: 6-7, 2014.

11. Facciorusso A and Barone M: Glucose intolerance and hepatocellular carcinoma: Recent findings for old diseases. Hepatobiliary Surg Nutr 3: 91-92, 2014.

12. He J, Zhang W, Li A, Chen F and Luo R: Knockout of NCOA5 impairs proliferation and migration of hepatocellular carcinoma cells by suppressing epithelial-to-mesenchymal transition. Biochem Biophys Res Commun 500: 177-183, 2018.

13. Sun K, Wang S, He J, Xie Y, He Y, Wang Z and Qin L: NCOA5 promotes proliferation, migration and invasion of colorectal cancer cells via activation of PI3K/AKT pathway. Oncotarget 8: 107932-107946, 2017.

14. Zheng ZC, Wang QX, Zhang W,Zhang XH and Huang DP: A novel tumor suppressor gene NCOA5 is correlated with progression in papillary thyroid carcinoma. OncoTargets Ther 11: 307-311, 2018.

15. Ye XH, Huang DP and Luo RC: NCOA5 is correlated with progression and prognosis in luminal breast cancer. Biochem Biophys Res Commun 482: 253-256, 2017.

16. Livak KJ and Schmittgen TD: Analysis of relative gene expression data using real-time quantitative PCR and the 2(-Delta Delta C(T)) method. Methods 25: 402-408, 2001.

17. Ranhotra HS: Estrogen-related receptor alpha and cancer: Axis of evil. J Recept Signal Transduct Res 35: 505-508, 2015.

18. Gao S, Li A, Liu F, Chen F, Williams M, Zhang C, Kelley Z, Wu CL, Luo R and Xiao H: NCOA5 haploinsufficiency results in glucose intolerance and subsequent hepatocellular carcinoma. Cancer Cell 24: 725-737, 2013.

19. Liang Y, Zhang T, Shi M, Zhang S, Guo Y, Gao J and Yang X: Low expression of NCOA5 predicts poor prognosis in human cervical cancer and promotes proliferation, migration, and invasion of cervical cancer cell lines by regulating notch 3 signaling pathway. J Cell Biochem 120: 6237-6249, 2019.

20. Chen GQ, Tian H, Yue WM, Li L, Li SH, Qi L, Gao C, Si LB and $\mathrm{Lu} \mathrm{M}$ : NCOA5 low expression correlates with survival in esophageal squamous cell carcinoma. Med Oncol 31: 376, 2014.

21. Wu Y, Wu J, Dong QR and Guo NZ: Association between expression of nuclear receptor co-activator 5 protein and prognosis in postoperative patients with osteosarcoma. Oncol Lett 15: 1888-1892, 2018.

22. Yuan X, Zhang L, Cui Y, Yu Y, Gao X and Ao J: NCOA5 is a master regulator of amino acid-induced mTOR activation and $\beta$-casein synthesis in bovine mammary epithelial cells. Biochem Biophys Res Commun 529: 569-574, 2020.

23. Li Y, Zhang T, Qin S, Wang R, Li Y, Zhou Z, Chen Y, Wu Q and Su F: Effects of UPF1 expression on EMT process by targeting E cadherin, $\mathrm{N}$ cadherin, Vimentin and Twist in a hepatocellular carcinoma cell line. Mol Med Rep 19: 2137-2143, 2019. 
24. Yamashita N, Tokunaga E, Iimori M, Inoue Y, Tanaka K, Kitao H, Saeki H, Oki E and Maehara Y: Epithelial Paradox: Clinical significance of coexpression of e-cadherin and vimentin with regard to invasion and metastasis of breast cancer. Clin Breast Cancer 18: e1003-e1009, 2018.

25. Paolillo $\mathrm{M}$ and Schinelli S: Extracellular matrix alterations in metastatic processes. Int J Mol Sci 20: 20, 2019.

26. Diepenbruck M and Christofori G: Epithelial-mesenchymal transition (EMT) and metastasis: Yes, no, maybe? Curr Opin Cell Biol 43: 7-13, 2016.

27. Chaffer CL, San Juan BP, Lim E and Weinberg RA: EMT, cell plasticity and metastasis. Cancer Metastasis Rev 35: 645-654, 2016.

28. Bill R and Christofori G: The relevance of EMT in breast cancer metastasis: Correlation or causality? FEBS Lett 589: 1577-1587, 2015.
29. Wong SHM, Fang CM, Chuah LH, Leong CO and Ngai SC: E-cadherin: Its dysregulation in carcinogenesis and clinical implications. Crit Rev Oncol Hematol 121: 11-22, 2018.

30. Zhang X, Liu G, Kang Y, Dong Z, Qian Q and Ma X: N-cadherin expression is associated with acquisition of EMT phenotype and with enhanced invasion in erlotinib-resistant lung cancer cell lines. PLoS One 8: e57692, 2013.

31. Guo L, Zhang K and Bing Z: Application of a co expression network for the analysis of aggressive and non aggressive breast cancer cell lines to predict the clinical outcome of patients. Mol Med Rep 16: 7967-7978, 2017. 